\title{
The investigation of thrombus formation process by Lyapunov's index of scattered coherent radiation
}

\author{
M.S.Gavrylyak ${ }^{1 *}$, S. G. Guminetsky ${ }^{1}$, P.M. Grygoryshyn ${ }^{2}$, P.P. Maksimyak ${ }^{1}$, M.V.Shaplavskyi ${ }^{2}$ \\ ${ }^{1}$ Correlation Optics Dept, Chernivtsi University, 2 Kotsyubinsky Str., Chernivtsi, 58012 Ukraine \\ ${ }^{2}$ Chernivtsi medical University, 2 Theatralna Str., Chernivtsi, 58000 Ukraine \\ mgavrylyak@gmail.com
}

\begin{abstract}
The results of experimental study of stochastization fluctuations of a coherent field intensity scattered by blood plasma are presented. It has been shown that time of thrombus formation can be defined by time dependence of Lyapunov' index of intensity fluctuations. It was discovered that time of thrombus formation of healthy people is smaller than ones sick by asthma.
\end{abstract}

Keywords: thrombus formation process, scattered laser radiation, stochastization

\section{INTRODUCTION}

In modern clinical and scientific researches the optical methods of investigation of components of system hemostasia gain wide application [1-3]. These methods register changes of optical properties of the incubatory environment during grume formation. The fibrinogen is the source of fibrin as in blood plasma. Investigation of the coagulation process of and fibrinolysis in plasma is of high interest for clinical practice as it gives the possibility of determination of concentration of fibrinogen under the conditions as much as possible approached to the physiology.

At present, the concentration of fibrinogen is determined by coagulation technique (by the time of grume formation in dissolved nonthrombocyte plasma after thrombin addition. Determination of thrombin time allows to estimate the final stage of fibrillation of a blood (a fibrinogen transformation into a fibrin).

The modern methods used for definition time thrombus formation have a number of shortcoming. To shortcoming refer high requirements to a transparency of plasma, dearness of the equipment, duration of research, etc. [3].

One of the most widespread methods of dynamic medium researching is the method dynamic light scattering [4]. Application of a dynamic light scattering method gives the possibility to carry out the researches which are not causing to change of medium properties, provide reception of objective data about medium and allow to fix with high time resolution change of structure of substance that is especially important in case of difficult physical and chemical and biological transients. Temporal fluctuations of intensity in a certain point of space caused by dynamic light scattering. They bear in themselves the information about investigated dynamic light scattering medium.

Application of the stochastic approach to the analysis of intensity fluctuations of a field scattered radiation gives the chance to solve partially a inverse problem that is important in cases of the dynamical medium connected with diagnostics of structural transformations.

The purpose of this work is the approbation of the method of thrombus formation time determination by the analysis of randomicity of scattered laser radiation by a blood plasma.

The Lyapunov's indices characterize the average speed of exponential divergence of close phase trajectories $[5,6]$. If $d_{0}$ is the initial distance between two initial dots of the phase trajectories, the distance between the trajectories outgoing from these dots at instant $t$ will be the following one:

$$
d(t)=d_{0} e^{\lambda t}
$$

The value $\lambda$ is called the Lyapunov's index. Each dynamic system is characterized by the spectrum of the Lyapunov's indices $\lambda i(i=1,2, \ldots, n)$, where $n$ - differential equations' number, which is necessary for describing the system. The availability of the positive value of the Lyapunov's index can be the proof of chaos existence in the system when the experimental data obtained during the investigation of dynamic systems. In general, the chaotic system is characterized by the divergence of phase trajectories at some directions and their convergence in the others. It means that there are either positive or negative Lyapunov's indices in the chaotic system. But for all that the sum of all parameters negative that is the 
degree of concurrence of trajectories exceeds the degree of divergence. If this condition is not carried out, the dynamic system is stable, and behavior of such system is easily recognized. Thus, in most cases, it is enough to calculate only the maximal Lyapunov's index. The positive value of the maximal Lyapunov's index proves the probability of chaos availability in the system, and this index magnitude characterizes the degree of randomicity.

\section{METHOTODOLOGY}

We investigated the degree of a randomness of scattered laser radiation by a blood plasma during the thrombus formation process. For experimental researches a blood of sick and healthy a people is used. Measurement of intensity fluctuations of scattered laser radiation by a blood plasma was carried out as follows: a cell with the sample (a blood plasma 0,2 $\mathrm{ml}$ ) was placed in accordance with the experiment scheme (fig. 1), and a solution of thrombin $(0,2 \mathrm{ml})$ was added. A thrombin causes transformation fibrinogen into fibrin, that causes fibrillation of a blood plasma. It is obvious, that such structural transformations influenced behavior of intensity fluctuations of scattered radiation. The recording of intensity fluctuations performed with the frequency of $50 \mathrm{kHz}$.

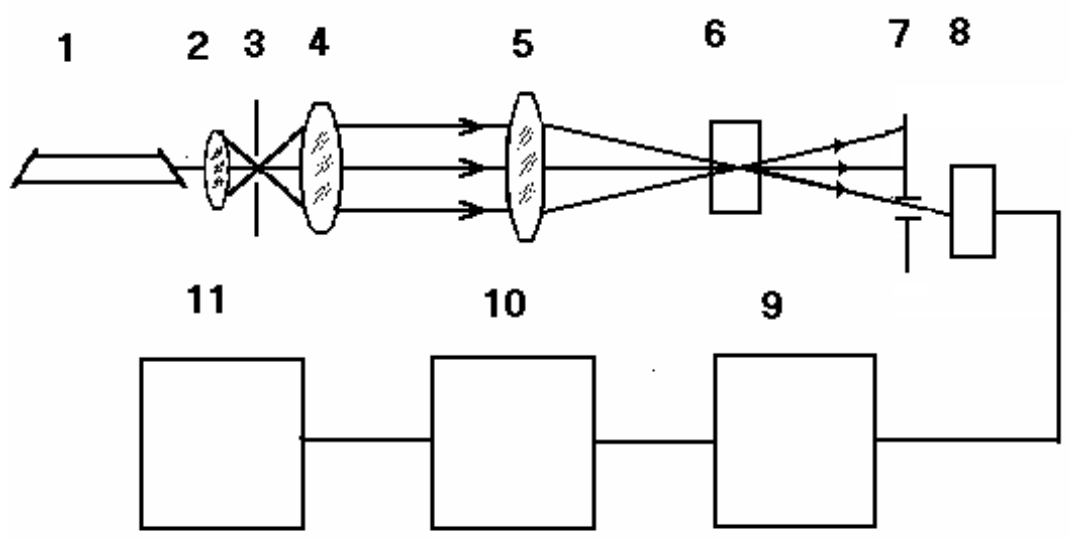

Fig.1. He-Ne laser beam is cleared of absent-minded beams by means of microlens 2, micrometer aperture 3, and lens 4. This wave is focused on a cell with whole blood 6 by means of a lens 5 . Photodetector 8 registers scattered radiation. The size of a diaphragm 7 should be less for a size of speckles in an absent-minded field. The computer 11 has an electrical signal from a photodetector through an operating amplifier 9 and through an analog-digital converter 10.

\section{RESULTS AND DISCUSION}

As a result of experiment, the time dependence of intensity of scattered laser radiation by blood plasma during thrombus formation has been found (fig. 2). The Lyapunov's index calculated on intervals much less for all time realization where the signal in the certain approach can be considered as statistically homogeneous, at consecutive shift of the analyzed dynamic series. Fig.3 demonstrates the time dependency of the calculated Lyapunov's indices. Addition a thrombin into a blood plasma (on 15 seconds from the data recording beginning (fig. 3)) has led to growth of randomicity of fluctuations of backscattered field of radiation, as a result of turbulent mixing thrombin with blood plasma. As a result of reaction of thrombin with fibrinogen threads of fibrin are formed. It causes the growth of viscosity of a blood plasma and reduction of randomicity of intensity fluctuations. The basic parameter of an estimation of time dependence maximum indicator of Lyapunov during thrombus formation process is: $\Delta \mathrm{t}_{1}$ - time of formation of a clot - a time difference between the position of a half-width of the dependence of the Lyapunov's index and beginning times of thrombus formation process). The half-width of the dependence of the Lyapunov's index selects on segment from position of a maximum till the end of the dependence. $\Delta \mathrm{t}$ - full time of formation of a clot. 


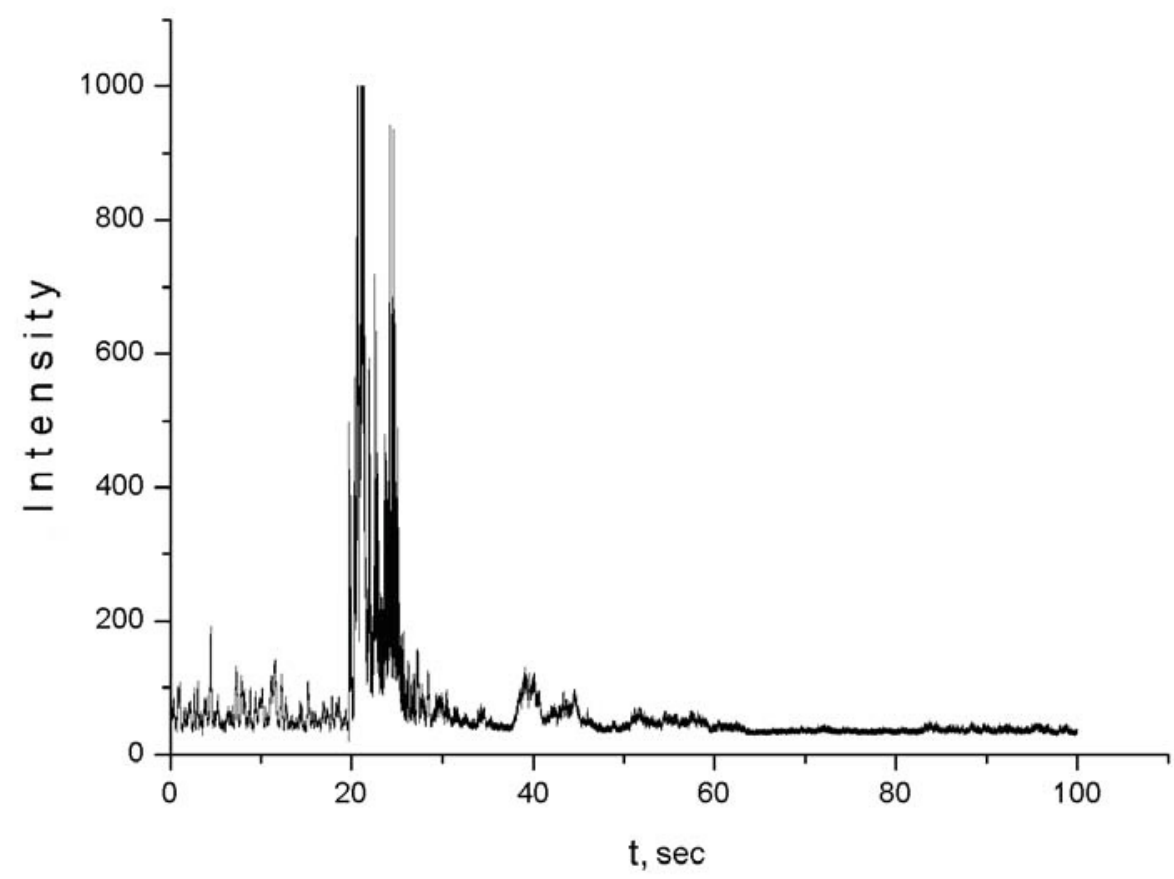

Fig.2. The intensity VS time dependence.

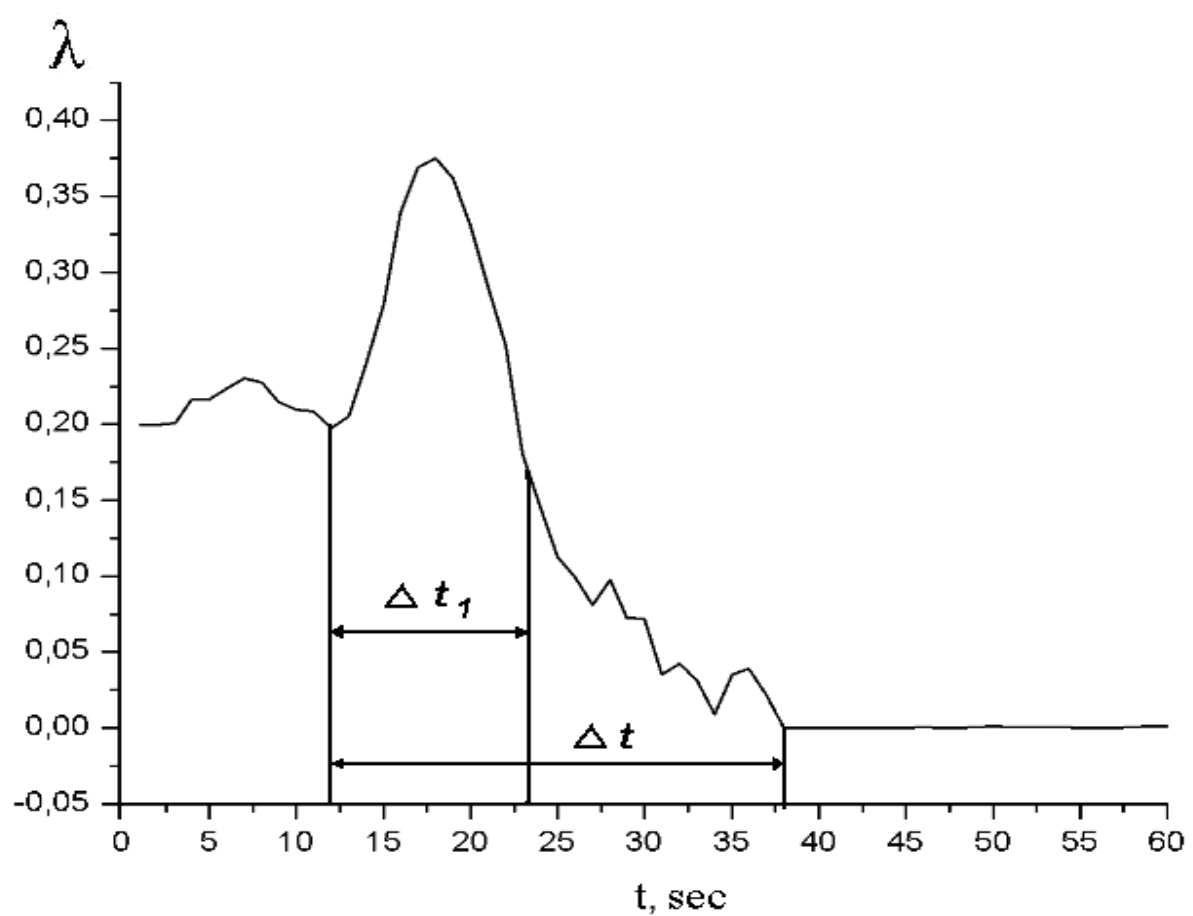

Fig.3. The time dependence of the Lyapunov's indices $\left(\Delta \mathrm{t}_{1}=12 \mathrm{sec}, \Delta \mathrm{t}=27 \mathrm{sec}\right)$.

For estimation of reliability of the received results we measured time thrombus formation for samples of plasma of the same blood by a turbidimetric method. The method is based on determination of magnitude of transmission coefficient of a blood plasma of a laser beam the wave length $(\lambda=633 i \imath)$ during the 
thrombin the addition. As a result of experiment, the time dependence of transmission coefficient of plasma in thrombus formation process is obtained (fig. 4). For turbidimetric method: $\Delta \mathrm{t}_{1}$ - time difference between the position of maximum of the dependence of transmission coefficient and beginning times of thrombus formation process. $\Delta \mathrm{t}-$ full time of formation of a clot.

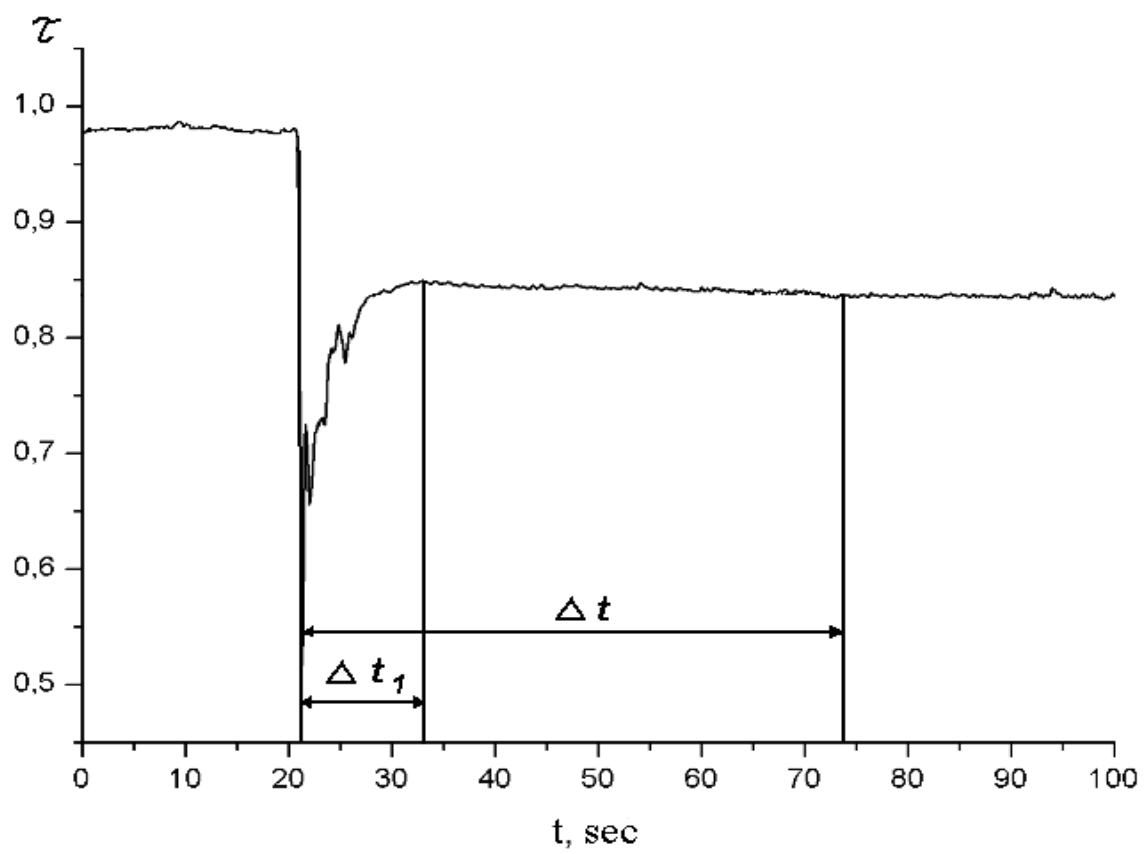

Fig.4. The time dependency of transmission coefficient of plasma $\left(\Delta \mathrm{t}_{1}=12 \mathrm{sec}, \Delta \mathrm{t}=52 \mathrm{sec}\right)$.

Analyzing the time dependence of the Lyapunov's indices (fig. 3) it is possible make a conclusion, that during action thrombin a stochastization of fluctuations of scattered radiation by a blood plasma decreases, that gives the possibility to estimate the time of formation of clot ( $\Delta t_{1}=12 \mathrm{sec}$.), which correlates in due course time of thrombus formation defined a turbidimetric method. Also, the proposed method gives the possibility to define full time of formation of a clot $(\Delta t=27 \mathrm{sec}$.) more accurately and unambiguous.

Using such technique the parameters thrombus formation blood healthy people and sick of asthma are investigated. It is revealed, that parameters thrombus formation $\Delta \mathrm{t}_{1}$ (time of formation of clot) and $\Delta \mathrm{t}$ (full time of formation of a clot) of healthy people are mainly more low (within one group of blood) than parameters thrombus formation of sick by asthma (tab. 1 - tab.4).

\begin{tabular}{|c|c|c|}
\hline People & $\overline{\Delta t}, \mathrm{sec}$ & $\overline{\Delta t}_{1}, \mathrm{sec}$ \\
\hline Healthy & 33 & 16 \\
\hline Sick & 41 & 25 \\
\hline
\end{tabular}

Tab.1. Parameters thrombus formation (blood group O).

\begin{tabular}{|c|c|c|}
\hline People & $\overline{\Delta t}, \mathrm{sec}$ & $\overline{\Delta t}_{1}, \mathrm{sec}$ \\
\hline Healthy & 43 & 17 \\
\hline Sick & 46 & 22 \\
\hline
\end{tabular}

Tab.2. Parameters thrombus formation (blood group A). 


\begin{tabular}{|c|c|c|}
\hline People & $\overline{\Delta t}$, sec & $\overline{\Delta t}_{1}, \mathrm{sec}$ \\
\hline Healthy & 31 & 12 \\
\hline Sick & 38 & 18 \\
\hline
\end{tabular}

Tab.3. Parameters thrombus formation (blood group B).

\begin{tabular}{|c|c|c|}
\hline people & $\overline{\Delta t}, \mathrm{sec}$ & $\overline{\Delta t}_{1}, \mathrm{sec}$ \\
\hline healthy & 40 & 15 \\
\hline sick & 48 & 24 \\
\hline
\end{tabular}

Tab.4. Parameters thrombus formation (blood group $A B$ ).

There are great number influencing thrombus formation parameters [1], therefore growth of thrombus formation parameters cannot be unique and singular criterion for a diagnosis of a bronchial asthma. But use of this fact as auxiliary symptom is very perspective.

\section{REFERENCES}

1. Sato H., Nakajima A. (1984) Thrombosis Research, 33, 645-651.

2. $\quad$ Carlson R.H., Garnick R.L., Jones A.J.S. (1988) Anal. Biochem., 168, 428-435.

3. MacFarlane R.G. An enzyme cascade in the blood clotting mechanism and its function as biochemical amplifier // Nature._- 1964._- Vol. 202._P. 498-499.

4. Kokhanovsky A. A. Optics of light scattering media: problems and solusolution. - John Wiley \& Son Ltd, 1999. - $228 \mathrm{p}$.

5. Yu. I. Neymark and P. S. Landa, Stochastic and Chaotic Oscillations, Nauka, Moscow, 1987, (in Russian).

6. Rosenstein M.T., Collins J.J., Luca Carlo J. De. A practical method for calculating largest Lyapunov exponents from small data sets (MA 02215, Boston University,1992). 